\title{
Equilibrio, cinética y termodinámica de la adsorción del colorante DB-86 sobre carbón activado de la cáscara de yuca
}

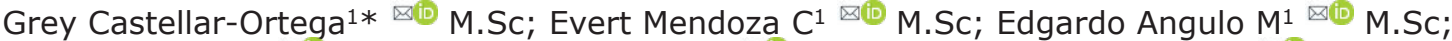

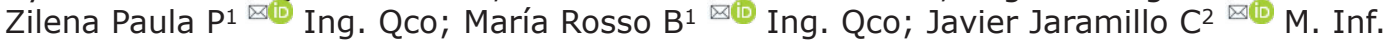

${ }^{1}$ Universidad del Atlántico, Facultad de Ciencias Básicas, Grupo de Investigación en Biotecnología de Microalgas, Fisicoquímica Aplicada y Estudios Ambientales, km 7 Antigua vía Puerto Colombia, Colombia.

2Universidad de la Costa, Departamento de Ciencias Básicas, Grupo de Investigación en Ciencias Naturales y Exactas, Barranquilla, Colombia.

*Correspondencia: greycastellar@mail.uniatlantico.edu.co

Recibido: Mayo 2018; Aceptado: Noviembre 2018; Publicado: Mayo 2019.

\section{RESUMEN}

Objetivo. Establecer mediante experimentos por lote la capacidad de remoción, la cinética y termodinámica de adsorción del carbón activado preparado a partir de la cáscara de yuca (Manihot esculenta) en la remoción del colorante azul directo 86. Materiales y métodos. La metodología experimental consistió inicialmente en la preparación del carbón activado por activación química de la cáscara de yuca con $\mathrm{H}_{3} \mathrm{PO}_{4}$ y su posterior calcinación a $530^{\circ} \mathrm{C}$. En la caracterización se determinaron las propiedades de textura mediante el índice de yodo e índice de azul de metileno, se cuantificaron los grupos funcionales orgánicos ácidos y básicos con el método Boehm, y se realizó el análisis próximo siguiendo las normas ASTM D-2867-70, ASTM D2866 y ASTM D2866-94. En el estudio por lote, el efecto de varios parámetros sobre la capacidad de adsorción fueron evaluados: el $\mathrm{pH}(2,4,8$ y 10$)$, la temperatura $\left(25,30\right.$ y $\left.40^{\circ} \mathrm{C}\right)$ y la concentración inicial de colorante $(20,40,60,80$ y $100 \mathrm{mg} / \mathrm{L})$. Tanto las características fisicoquímicas como los ensayos de adsorción del carbón activado preparado a partir de la cáscara de yuca (CAY) fueron comparadas con otro de marca comercial (CAM). Resultados. Los resultados de la caracterización indican que ambos carbones tienen una química de superficie heterogénea, de naturaleza ácida para el CAY y básica para el CAM. La máxima capacidad obtenida fue $6.1 \mathrm{mg} / \mathrm{g}$ para el CAY y de $3.7 \mathrm{mg} / \mathrm{g}$ para el CAM. Los cálculos termodinámicos indican que la remoción es espontánea y para ambos carbones la cinética se ajusta al modelo de pseudo segundo orden. Conclusiones. El carbón activado obtenido a partir de la cáscara de yuca puede considerarse un adsorbente eficiente en la remoción de colorantes.

Palabras clave: Adsorción, carbón activado, colorante azul directo 86, contaminantes (Fuente: CAB).

\begin{abstract}
Objective. To establish by means of experimenting by batch the capacity of removal, the kinetics and adsorption thermodynamics of activated carbon prepared from manioc husk (Manihot esculenta) in the removal of direct blue 86 dye. Materials and methods. Firstly, the experimental methodology worked on the preparation of activated carbon by chemical activation of manioc husk with $\mathrm{H}_{3} \mathrm{PO}_{4}$ calcined at $530^{\circ} \mathrm{C}$. In the characterization the texture properties were determined by means of the blue methylene and iodine indices, the basic and acidic functional groups were quantified by the Boehm method, and the proximate analyses were done following the norms ASTM D2867-70, ASTM D2866 and ASTM D2866-94. During the batch studies, the effect of several parameters over the adsorption capacity was evaluated: $\mathrm{pH}(2,4,8$ and 10$)$, temperature $\left(25,30\right.$ and $\left.40^{\circ} \mathrm{C}\right)$ and initial concentration of the dye $(20,40,60$, 80 and $100 \mathrm{mg} / \mathrm{L}$ ). Both physicochemical and adsorption characteristics of the activated carbon from manioc husk (CAY) were compared against those of a commercial brand (CAM). Results. The results of characterization showed that both carbons have a chemistry heterogeneous surface, acidic for CAY and basic for CAM. The maximum capacity obtained was $6.1 \mathrm{mg} / \mathrm{g}$ for CAY and $3.7 \mathrm{mg} / \mathrm{g}$ for CAM. The thermodynamic calculations showed that the removal was spontaneous. The kinetics for both carbon samples fits a pseudo second-order model. Conclusions. The activated carbon obtained from the manioc husk can be considered an efficient adsorbent for the removal of dyes.
\end{abstract}

Keywords: Activated carbon, adsorption, direct blue 86 dye, pollutant (Source: $C A B$ ).

Como citar (Vancouver)

Castellar-Ortega G, Mendoza CE , Angulo ME, Paula PZ, Rosso BM, Jaramillo CJ. Equilibrio, cinética y termodinámica de la adsorción del colorante DB-86 sobre carbón activado de la cáscara de yuca. Rev MVZ Cordoba. 2019; 24(2):7231-7238. DOI: https://doi.org/10.21897/rmvz.1700

(C)EI (los) autor (es), Revista MVZ Córdoba 2019. Este artículo se distribuye bajo los términos de la licencia internacional Creative Commons Attribution 4.0 (https://creativecommons.org/licenses/by-sa/4.0/), que permite el uso sin restricciones, la distribución y la reproducción en cualquier medio, siempre que se otorgue el crédito apropiado al autor o autores originales y la fuente. 


\section{INTRODUCCIÓN}

Las industrias como la textil, cuero, imprenta, cosmética, productos farmacéuticos, plásticos y alimentos utilizan diferentes tipos de colorantes que también aparecen en las aguas residuales descargadas por algunas de estas industrias (1-3). La presencia de color en los cuerpos de agua incide sobre la diversidad acuática porque impide la penetración de la luz solar afectando la fotosíntesis considerablemente $(1,4)$, además, se conoce que estos compuestos contienen aminas aromáticas, bencidina y otros compuestos aromáticos tóxicos, carcinógenos y mutagénicos para los seres humanos $(3,5,6)$.

Los colorantes azoicos representan la clase más grande y versátil de los colorantes sintéticos. Contienen uno o más enlaces azo $(-\mathrm{N}=\mathrm{N}-)$ como grupo cromóforo en asociación con estructuras complejas que contienen otros grupos funcionales tales como $-\mathrm{NH}_{2},-\mathrm{SO}_{3},-\mathrm{OH}(3,7,8)$. Además, estos colorantes pueden formar fuertes compuestos de coordinación con iones de metales de transición, como $\mathrm{Ni}$ (II), Cr (III), Co (II) y Cu (II) entre otros, formando quelatos azo-metal estables que no pueden metabolizarse ni degradarse $(2,9)$.

Existen varias técnicas para la eliminación de colorantes de las aguas residuales. Por los resultados obtenidos y su versatilidad en la remoción de diferentes tipos de colorantes, se considera que la adsorción es una de las mejores $(10,11)$. La mayoría de los sistemas comerciales actualmente utiliza carbón activado como adsorbente para eliminar colorantes debido a su alta área superficial y volumen de poro (12), excelente capacidad de adsorción, rápida y fácil regeneración (13). Quizás uno de los principales inconvenientes de su empleo es el uso de precursores no renovables como el carbón mineral y la madera, relativamente costosos. En este sentido, las investigaciones se han enfocado hacia la producción de carbón activado usando precursores no convencionales, materiales renovables, abundantes y de bajo costo, como la biomasa proveniente de desechos agrícolas e industriales (14-16).

Bajo este contexto, en esta investigación se preparó carbón activado por activación química con $\mathrm{H}_{3} \mathrm{PO}_{4}$ empleando cáscara de yuca como precursor. Se evaluaron sus características fisicoquímicas, su capacidad de adsorción, termodinámica y cinética en la remoción del colorante azul directo 86 (DB-86) y, además, se comparó con otro de marca comercial. Los datos experimentales fueron ajustados a modelos matemáticos de isotermas de adsorción y cinéticos.

\section{MATERIALES Y MÉTODOS}

Preparación del carbón activado. La cáscara de yuca se lavó con abundante agua para retirar la tierra y material no deseable, se secó durante 3 días al sol y después por 24 h a $105^{\circ} \mathrm{C}$ (17) en un horno de convección forzada marca Esco Isotherm ${ }^{\circledR}$ OFA 32TN-9. Una vez seca la cáscara, se trituró en un molino marca MACSA 300 monitor 525V con impulsión $4 \mathrm{~kW}$, hasta alcanzar el tamaño de partícula entre 1 y $4 \mathrm{~mm}$ (granulometría de 5x18, U. S. Standard Sieve). El material molido se lavó con agua desionizada y se secó en un horno por $24 \mathrm{~h}$ a $105^{\circ} \mathrm{C}$. La activación química de este precursor consistió en adicionar $1 \mathrm{~mL}$ de disolución de $\mathrm{H}_{3} \mathrm{PO}_{4}$ al $85 \%$ (18) por cada gramo de cáscara molida y seca, esta mezcla se mantuvo en contacto por $24 \mathrm{~h}$ a $25^{\circ} \mathrm{C}$. Luego se calcinó en una mufla durante $30 \mathrm{~min}$ a $530^{\circ} \mathrm{C}$. Por último, el carbón activado obtenido se dejó enfriar y se lavó con $\mathrm{HCl}$ concentrado durante $6 \mathrm{~h}$ en agitación continua, para eliminar fosfatos y otras impurezas. Pasado este tiempo se lavó con agua desionizada hasta conseguir un $\mathrm{pH}$ cercano a 7, empleando un medidor de $\mathrm{pH}$ marca Orion StarTM A221, se secó por $1 \mathrm{~h}$ a $105^{\circ} \mathrm{C}$ y se tamizó hasta conseguir un tamaño de partícula entre 0.500 a 1.680 $\mathrm{mm}$ (granulometría de 12×35, U. S. Standard Sieve). Para el caso del carbón activado comercial marca Merck, este solo se lavó con suficiente agua desionizada y se secó en un horno a $105^{\circ} \mathrm{C}$ durante $24 \mathrm{~h}$.

Caracterización de los carbones activados. Es de vital importancia evaluar ciertas propiedades físicas y químicas del carbón activado, de esta manera, se puede lograr una mejor interpretación de los mecanismos que se involucran durante los procesos que puedan darse. Para ello, se realizó el análisis próximo de los diferentes adsorbentes, el cual incluye contenido de humedad, cenizas y material volátil, siguiendo las normas ASTM D-2867-70, ASTM D2866 y ASTM D2866-94 respectivamente. El tamaño de poros se determinó por dos métodos: por medio del índice de yodo siguiendo la norma ASTM D460794 y, por adsorción con azul de metileno aplicando el método Chemviron Carbon Company, el cual consistió en adicionar una cantidad de carbón activado a un volumen de disolución estándar de azul de metileno. La capacidad máxima $\left(\mathrm{q}_{\max }\right)$ se calculó a partir de la ecuación 1 .

$\mathrm{q}_{\max }(\mathrm{mg} / \mathrm{g})=\frac{\left(\mathrm{C}_{\mathrm{o}}-\mathrm{C}_{\mathrm{e}}\right) \mathrm{V}}{\mathrm{M}}[1]$

Donde $\mathrm{C}_{\mathrm{o}}$ y $\mathrm{C}_{\mathrm{e}}$ son las concentraciones iniciales y en el equilibrio del colorante en $\mathrm{mg} / \mathrm{L}, \mathrm{V}$ es el volumen de disolución en litros y $M$ es la masa de carbón activado empleada en gramos. El punto de carga cero (PCC) se determinó colocando en varios frascos $0.5 \mathrm{~g}$ de adsorbente en $50 \mathrm{~mL}$ de agua desionizada, luego se ajustó el $\mathrm{pH}$ entre 3 y 12 unidades con soluciones de $\mathrm{HCl} 0.1 \mathrm{M}$ y $\mathrm{NaOH} 0.1 \mathrm{M}$. Transcurridas $48 \mathrm{~h}$ se midió el pH final. Por último, se realizó el método de Boehm para cuantificar los grupos funcionales ácidos y básicos orgánicos presentes $(19,20)$. Esta prueba consistió en adicionar $1 \mathrm{~g}$ de carbón activado a $100 \mathrm{~mL}$ de disolución de $\mathrm{NaOH} 0.1 \mathrm{M}, \mathrm{Na}_{2} \mathrm{CO}_{3} 0.1 \mathrm{M}, \mathrm{NaHCO}_{3} 0.1 \mathrm{M}$ y HCl 0.1 $\mathrm{M}$ por $24 \mathrm{~h}$, cada una por separado. Por último, se tomó una alícuota y se tituló con disoluciones estándar de $\mathrm{NaOH}$ y $\mathrm{HCl}$ según el caso. Todos los reactivos químicos usados fueron grado analítico marca Merck ${ }^{\circledR}$.

Estudio por lote, termodinámico y cinético. Inicialmente, se preparó una disolución "stock" de 1000 mg/L del colorante azul directo 86 , colorante tipo ftalocianina de cobre, de fórmula molecular $\mathrm{C}_{32} \mathrm{H}_{14} \mathrm{CuN}_{8} \mathrm{Na}_{2} \mathrm{O}_{6} \mathrm{~S}_{2}$ (Figura 1), a partir de esta disolución se prepararon varias diluciones de $20,40,60,80$ y $100 \mathrm{mg} / \mathrm{L}$. A $50 \mathrm{~mL}$ de cada una de las anteriores diluciones se le adicionó $1 \mathrm{~g}$ de carbón activado, se ajustó el pH a 2 con $\mathrm{HCl}$ diluido (seleccionado del estudio de efecto del $\mathrm{pH}$ ) y se colocaron en un agitador horizontal a $120 \mathrm{rpm}$ por $12 \mathrm{~h}$. Por último, se filtró, se tomó una alícuota y se le determinó la concentración en un espectrofotómetro UV-vis marca Spectronic Genesys 20, a una longitud de onda de $615 \mathrm{~nm}$, previamente determinada por barrido. Cada uno de los anteriores experimentos se realizó por triplicado y a diferentes temperaturas $\left(25,30\right.$ y $\left.40^{\circ} \mathrm{C}\right)$. La capacidad de adsorción y el porcentaje de remoción se calcularon a partir de las ecuaciones 1 y 2 . Para el estudio cinético se seleccionaron las condiciones de concentración inicial de colorante de $100 \mathrm{mg} / \mathrm{L}$ y $\mathrm{pH} 2$ y, a diferencia del procedimiento anterior se tomaron alícuotas entre los 60 y $720 \mathrm{~min}$.

$\% R=\frac{\left(C_{o}-C_{e}\right)}{C_{o}} \times 100 \%[2]$ 


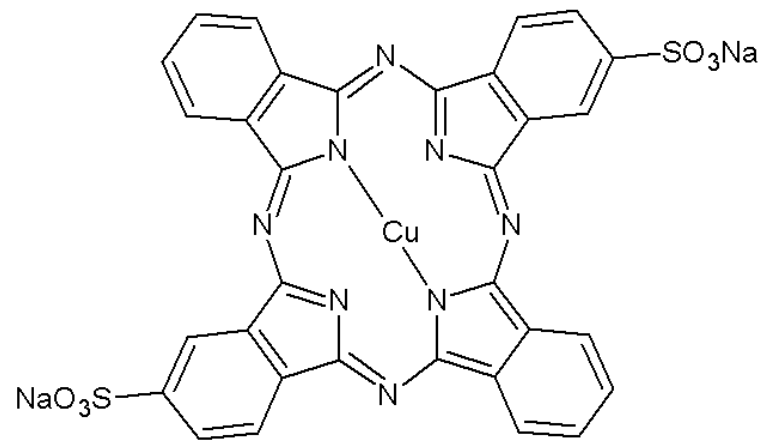

Figura 1. Estructura molecular del colorante DB-86.

Para evaluar el efecto del $\mathrm{pH}$ sobre la capacidad de adsorción se realizó un procedimiento muy similar al mencionado anteriormente. Este consistió en preparar una dilución de $100 \mathrm{mg} / \mathrm{L}$, ajustándole el $\mathrm{pH}$ con $\mathrm{HCl}$ y $\mathrm{NaOH}$ diluido hasta lograr valores de 2, 4, $810 \pm 0.1$, a la temperatura del laboratorio $\left(25^{\circ} \mathrm{C}\right)$.

\section{RESULTADOS}

Caracterización fisicoquímica. La curva experimental de cada uno de los adsorbentes para la determinación del potencial de carga cero (PCC) se muestran en la Figura 2. Los valores de PCC para el carbón activado obtenido a partir de la cáscara de yuca y para el carbón comercial, son respectivamente 3.1 y 4.2 . Esto implica que aquellos adsorbentes con valores de $\mathrm{pH}$ mayores que el PCC tienen una superficie con predominio de cargas negativas, mientras que, un $\mathrm{pH}$ menor que el PCC se tendrá una superficie con predominio de cargas positivas.
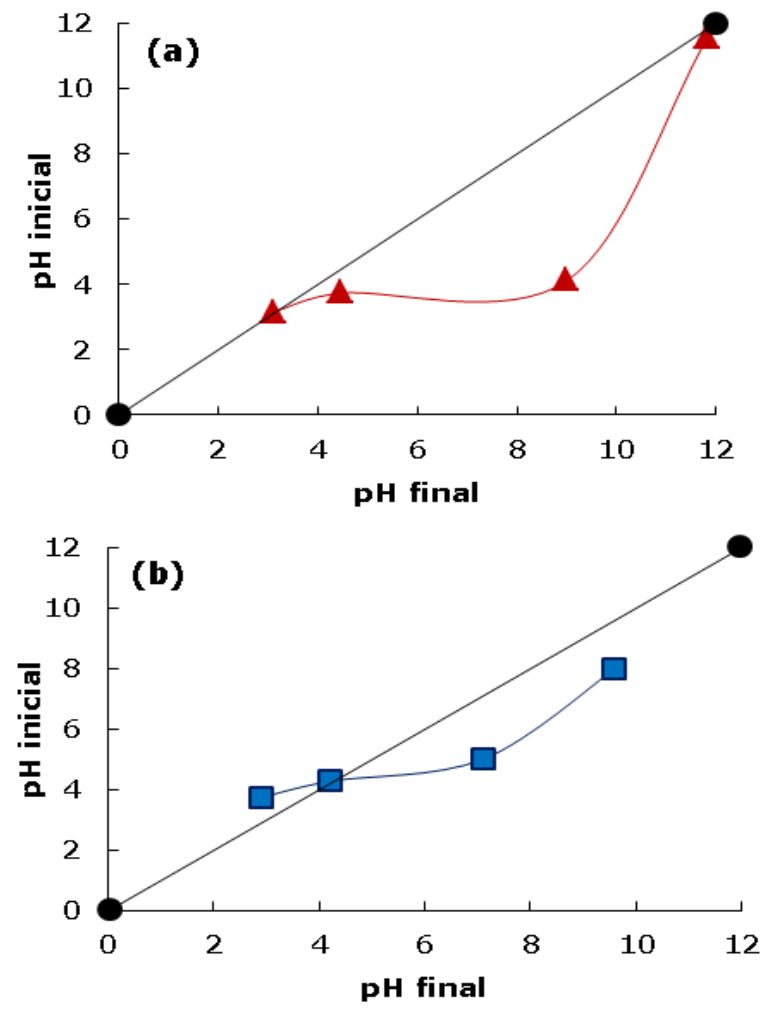

Figura 2. Punto de carga cero del CAY (a) y CAM (b).
En la tabla 1 se muestra comparativamente las características fisicoquímicas de los carbones activados CAY y CAM. Del análisis próximo se observa que el porcentaje de humedad y de materia volátil es menor en el carbón activado preparado con cáscara de yuca, en comparación con el carbón comercial. Por otro lado, el porcentaje de cenizas, aunque bajo para ambos carbones activados, presenta un ligero aumento en el CAY. Se sugiere que el mayor contenido de humedad en el carbón activado CAM (17.4\%), se debe principalmente a las condiciones ambientales y de almacenamiento del mismo, al tratarse de un sólido poroso adsorbente retiene la humedad del aire en su superficie. Con respecto a las propiedades de textura: área específica, volumen de microporos y volumen total de poros, estos se calcularon a partir del índice de yodo e índice de azul de metileno. El índice de yodo es una medida de la adsorción de yodo en una solución acuosa, es una medida de microporos y se utiliza como una estimación de la superficie total.

Tabla 1. Resultados de La caracterización fisicoquímica de los adsorbentes.

\begin{tabular}{lcc}
\hline \multirow{2}{*}{ Propiedad } & \multicolumn{2}{c}{ Adsorbente } \\
\cline { 2 - 3 } & CAY & CAM \\
\hline Análisis próximo & 13.1 & 17.4 \\
Humedad (\%) & 5.2 & 3.2 \\
Cenizas (\%) & 94.8 & 96.8 \\
Materia volátil (\%) & 62.4 & 178.5 \\
Índice de azul de metileno (mg/g) & 890 & 595 \\
Índice de yodo (mg/g) & 472 & 334 \\
Área específica (m $/ \mathrm{g})$ & 0.23 & 0.22 \\
Volumen de microporos (cm $\left.{ }^{3} / \mathrm{g}\right)$ & 0.34 & 0.54 \\
Volumen total de poros (cm $3 / \mathrm{g})$ & & \\
Grupos funcionales ácidos & 0.06 & 0.0 \\
Fenólicos (meq/g) & 0.0 & 0.06 \\
Lactónicos (meq/g) & 0.62 & 0.19 \\
Carboxílicos (meq/g) & 0.68 & 0.25 \\
Acidez total (meq/g) & 0.0 & 0.56 \\
\hline \multicolumn{1}{c}{ Basicidad total (meq/g) } & &
\end{tabular}

Adsorbentes con alto índice de yodo tiene un mejor rendimiento en la eliminación de contaminantes de tamaño pequeño, en tanto que, el índice de azul de metileno muestra tamaños mayores de poro desarrollados durante la activación. Es importante resaltar que para el cálculo del valor del índice de yodo, se tomaron muestras de $1.2 \mathrm{~g}$ de CAY y $1.8 \mathrm{~g}$ de CAM, para que el factor de corrección tabulado del índice de yodo se ajustara a cada muestra de carbón analizada. En la tabla 1 se observa que el carbón activado CAY logró desarrollar mayor área específica $\left(472 \mathrm{~m}^{2} / \mathrm{g}\right)$ con predominio de microporos que favorecen el proceso de adsorción. Con respecto a la estimación de poros de mayor tamaño, el resultado del índice de azul de metileno muestra que el carbón CAM, con una capacidad de adsorción de $178.5 \mathrm{mg} / \mathrm{g}$ es el que posee más estructuras macro y mesoporosas.

Asumiendo que el $\mathrm{NaOH}$ neutraliza a los grupos carboxílicos, lactónicos y fenólicos; el $\mathrm{Na}_{2} \mathrm{CO}_{3}$ a los grupos carboxílicos y lactónicos, el $\mathrm{NaHCO}_{3}$ solo a los grupos carboxílicos y el $\mathrm{HCl}$ a los grupos básicos, se realizaron los cálculos correspondientes para determinar la cantidad en $\mathrm{meq} / \mathrm{g}$ de estos grupos funcionales para los dos carbones activados. En la tabla 1 se resumen los resultados. 
Estudio por lote, termodinámico y cinético. La figura 3 muestra como disminuye la capacidad de adsorción de los carbones activados evaluados cuando se aumenta el $\mathrm{pH}$ de la solución a $25^{\circ} \mathrm{C}$. A valores bajos de pH (2 a 4) la concentración de iones hidronio $\left(\mathrm{H}^{+}\right)$aumenta, cargándose positivamente la superficie del carbón activado, situación que favorece las atracciones electrostáticas con las moléculas del colorante DB-86 de naturaleza aniónica. La capacidad máxima de adsorción para ambos carbones activados se alcanzó a pH 2, razón por la cual, se seleccionó este pH.

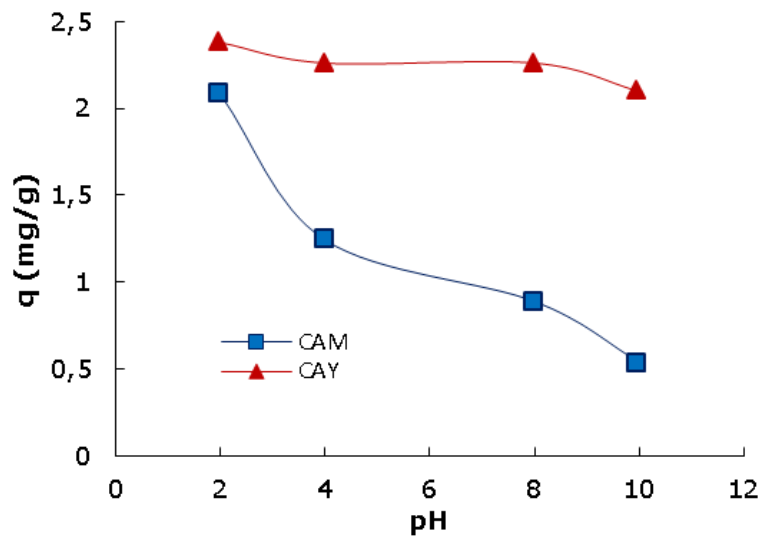

Figura 3. Efecto del pH sobre la capacidad máxima de adsorción.

Del estudio por lote se obtuvieron las isotermas de adsorción, estas indican cómo las moléculas del adsorbente se distribuyen entre las fases líquida y sólida cuando el proceso alcanza el estado de equilibrio. Los datos obtenidos se ajustaron a los modelos de isotermas Langmuir y Freundlich. Las ecuaciones linealizadas de ambos modelos se representan mediante las ecuaciones 3 y 4 respectivamente.

$$
\begin{aligned}
& \frac{\mathrm{C}_{\mathrm{e}}}{\mathrm{q}_{\mathrm{e}}}=\frac{1}{\mathrm{~K}_{\mathrm{L}} \mathrm{q}_{\max }}+\frac{\mathrm{C}_{\mathrm{e}}}{\mathrm{q}_{\max }} \text { [3] } \\
& \log \mathrm{q}_{\mathrm{e}}=\log \mathrm{K}_{\mathrm{f}}+\left(\frac{1}{n}\right) \log \mathrm{C}_{\mathrm{e}} \text { [4] }
\end{aligned}
$$

Donde $q_{e}(\mathrm{mg} / \mathrm{g})$ es la capacidad máxima de adsorción del adsorbente, $\left(\mathrm{dm}^{3} / \mathrm{mg}\right) \mathrm{K}_{\mathrm{L}}$ es la constante de Langmuir y está relacionada con el calor de adsorción, $\mathrm{K}_{\mathrm{f}}(\mathrm{mg} / \mathrm{g}$ $\left(\mathrm{dm}^{3} / \mathrm{mg}\right)^{1 / \mathrm{n}}$ ) es la constante de Freundlich, $\mathrm{n}$ es una constante que indica la intensidad de la adsorción y $C_{e}\left(\mathrm{mg} / \mathrm{dm}^{3}\right)$ es la concentración en el equilibrio. Estos parámetros proporcionan información importante sobre los mecanismos de adsorción, las propiedades de superficie y afinidades del adsorbente. En la tabla 2 se registran los valores obtenidos por regresión lineal.

Tabla 2. Parámetros de adsorción para las isotermas de Langmuir y Freundlich.

\begin{tabular}{ccccccccc}
\hline & & \multicolumn{3}{c}{ Constantes de Langmuir } & \multicolumn{4}{c}{ Constantes de Freundlich } \\
\cline { 3 - 8 } & $\mathbf{T}\left({ }^{\circ} \mathbf{C}\right)$ & $\begin{array}{c}\mathbf{q}_{\max } \\
(\mathbf{m g} / \mathbf{g})\end{array}$ & $\mathbf{K}_{\mathbf{L}}(\mathbf{L} / \mathbf{m g})$ & $\boldsymbol{R}^{2}$ & $\mathbf{n}$ & $\begin{array}{c}\mathbf{K}_{\mathbf{f}} \\
\left(\mathbf{m g} / \mathbf{g}(\mathbf{L} \mathbf{m g})^{\mathbf{1} / \mathbf{n}}\right)\end{array}$ & $\boldsymbol{R}^{\mathbf{2}}$ \\
\hline CAM & 25 & 3.1 & 0.015 & 0.86 & 1.60 & 0.116 & 0.97 \\
CAM & 30 & 3.6 & 0.032 & 0.91 & 1.75 & 0.246 & 0.99 \\
CAM & 40 & 3.7 & 0.036 & 0.94 & 1.81 & 0.277 & 0.99 \\
CAY & 25 & 6.2 & 0.008 & 0.56 & 1.22 & 0.080 & 0.94 \\
CAY & 30 & 3.8 & 0.029 & 0.87 & 1.66 & 0.223 & 0.98 \\
CAY & 40 & 3.9 & 0.033 & 0.72 & 1.72 & 0.260 & 0.93 \\
\hline
\end{tabular}

$\mathrm{AD}=$ Adsorbente; $\mathrm{T}=$ Temperatura;
Las propiedades termodinámicas como el cambio de energía de Gibbs $\left(\Delta \mathrm{G}^{\circ}\right)$, de entalpía $\left(\Delta \mathrm{H}^{\circ}\right)$ y de entropía $\left(\Delta \mathrm{S}^{\circ}\right)$ se estimaron con el fin de comprender la viabilidad y la naturaleza del proceso de adsorción (21). A partir de la constante aparente de equilibrio definida en la ecuación 3 del modelo de isoterma de Langmuir, se calculó el cambio en la energía de Gibbs, $\Delta \mathrm{G}^{\circ}(\mathrm{kJ} /$ mol), para cada una de las temperaturas evaluadas $T$ (K), empleando la ecuación 5, donde $\mathrm{R}$ es la constante universal de los gases $(8.314 \mathrm{~J} / \mathrm{molK})$.

$\Delta \mathrm{G}^{\circ}=-\mathrm{RT} \ln \mathrm{K}_{\mathrm{L}}[5]$

El cambio en la energía de Gibbs indica el grado de espontaneidad del proceso, valores negativos reflejan una mayor favorabilidad de la adsorción y su variación puede expresarse en función del cambio en la entalpia $\Delta \mathrm{H}^{\circ}(\mathrm{kJ} / \mathrm{mol})$, en la entropía $\Delta \mathrm{S}^{\circ}(\mathrm{J} / \mathrm{molK})$ y la temperatura, como lo indica la ecuación 6.

$\Delta \mathrm{G}^{\circ}=\Delta \mathrm{H}^{\circ}-\mathrm{T} \Delta \mathrm{S}^{\circ}[6]$

Al remplazar la ecuación 5 en la 6 resulta la siguiente expresión:

$\ln \mathrm{K}_{\mathrm{L}}=\frac{\Delta \mathrm{S}^{\circ}}{\mathrm{R}}-\frac{\Delta \mathrm{H}^{\circ}}{\mathrm{RT}}[7]$

La figura 4 muestra la representación de $\ln \left(K_{L}\right)$ en función de $1 / T$, donde la pendiente y el intercepto resultante de la gráfica son utilizados para determinar $\Delta \mathrm{H}^{\circ}$ y $\Delta \mathrm{S}^{\circ}$ respectivamente. Los valores de las propiedades termodinámicas se muestran en la tabla 3.

Con respecto a la cinética de adsorción los resultados experimentales fueron ajustados a los modelos cinéticos de pseudo primer orden y pseudo segundo orden. Las ecuaciones 8 y 9 muestran sus expresiones matemáticas linealizadas.

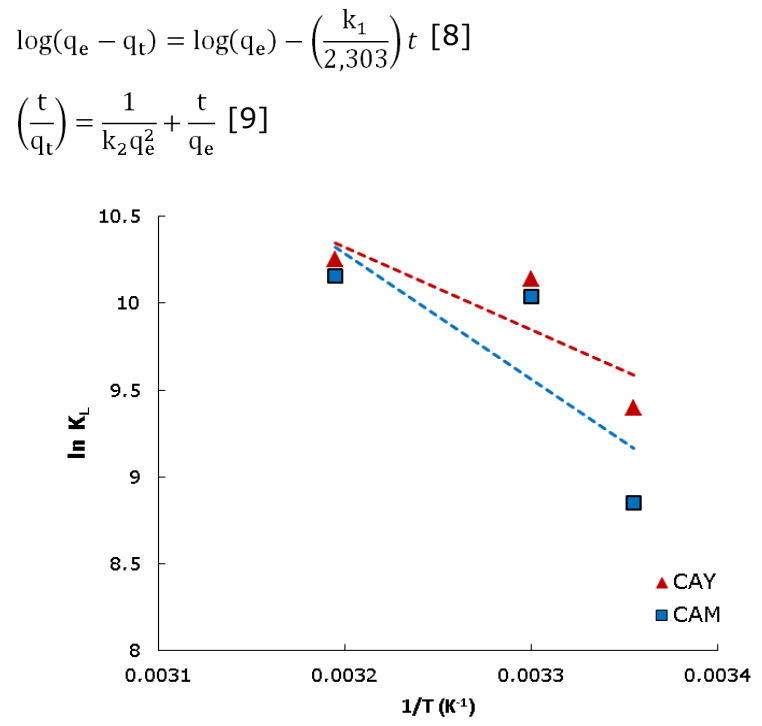

Figura 4. Termodinámica de la adsorción del colorante DB-86 sobre los carbones activados CAY y CAM a pH 2.

Donde $\mathrm{q}_{\mathrm{e}}$ y $\mathrm{q}_{\mathrm{t}}(\mathrm{mg} / \mathrm{g})$ son la capacidad de adsorción en el equilibrio y en un tiempo $t$ respectivamente $y_{1} k_{1}\left(m^{-1}\right)$ y $\mathrm{k}_{2}(\mathrm{~g} / \mathrm{mgmin})$ son las constantes de velocidad para cada modelo $(4,22)$. Los valores de $q_{e} y k_{1}$ fueron calculados de las gráficas lineales del $\log \left(\mathrm{q}_{\mathrm{e}}-\mathrm{q}_{\mathrm{t}}\right)$ versus $\mathrm{t}$ como se muestra en la figura 5. De igual manera, Los valores de $k_{2}$ y $q_{e}$ se calcularon a partir de la pendiente e intercepto de la línea recta que resulta de graficar graficar $t / q_{t}$ contra $t$ (Figura 6 ). Los valores de todas las constantes a diferentes temperaturas para los carbones CAY y CAM se muestran en la tabla 4. 
Tabla 3. Parámetros termodinámicos de la adsorción del colorante DB-86.

\begin{tabular}{cccc}
\hline \multirow{2}{*}{$\mathbf{T}\left({ }^{\circ} \mathbf{C}\right)$} & \multirow{2}{*}{ Adsorbente } & \multicolumn{2}{c}{ Parámetro termodinámico } \\
\cline { 3 - 4 } & & $\mathbf{K}_{\mathbf{L}}(\mathbf{L m o l})$ & $\mathbf{\Delta G}^{\circ}(\mathbf{k J} / \mathbf{m o l})$ \\
\hline 25 & CAM & 12082 & -23.3 \\
30 & CAM & 25399 & -25.5 \\
40 & CAM & 28316 & -26.7 \\
25 & CAY & 6977 & -21.9 \\
30 & CAY & 22877 & -25.3 \\
40 & CAY & 25928 & -26.4 \\
\hline Adsorbente & $\mathbf{\Delta H}^{\circ}(\mathbf{k J} / \mathbf{m o l})$ & $\mathbf{\Delta S}^{\circ}(\mathbf{J} / \mathbf{m o l K})$ & $\mathbf{R}^{\mathbf{2}}$ \\
\hline CAM & 39.5 & 212.4 & 0.70 \\
CAY & 60.5 & 279.1 & 0.67 \\
\hline
\end{tabular}

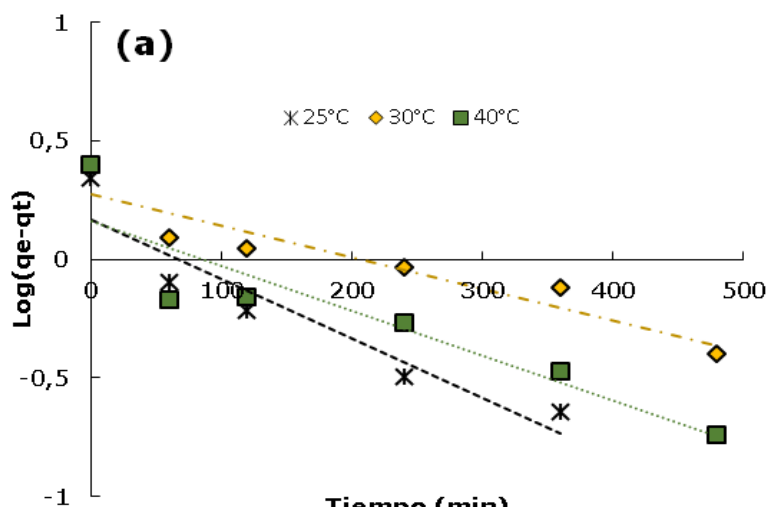

Tiempo (min)

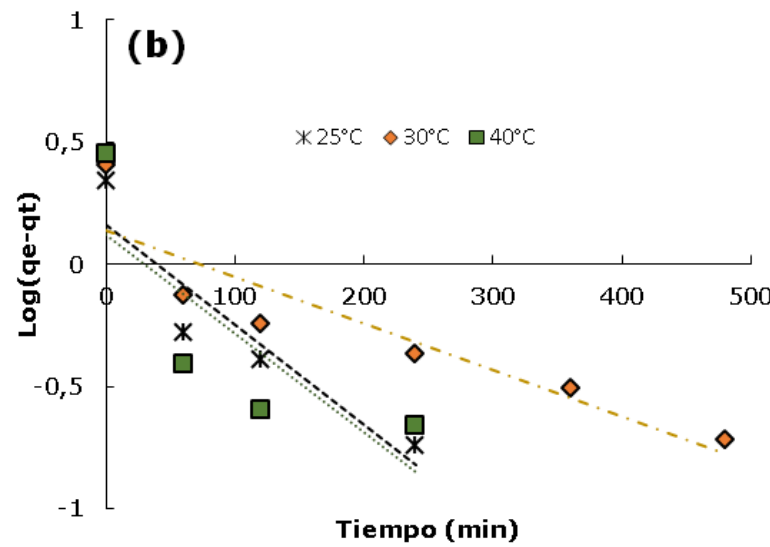

Figura 5. Modelo cinético pseudo primer orden para la adsorción del colorante DB-86 a diferentes temperaturas: a) CAM y b) CAY.

Tabla 4. Constantes cinéticas para los modelos de pseudo primer y pseudo segundo orden.

\begin{tabular}{|c|c|c|c|c|c|c|c|c|}
\hline \multirow[b]{2}{*}{$T\left({ }^{\circ} \mathrm{C}\right)$} & \multirow[b]{2}{*}{ AD } & \multirow{2}{*}{$\begin{array}{c}q_{e} \\
(\exp ) \\
(\mathrm{mg} / \mathrm{g}\end{array}$} & \multicolumn{3}{|c|}{ Constantes PP } & \multicolumn{3}{|c|}{ Constantes PS } \\
\hline & & & $\begin{array}{c}k_{1} \\
(L / \mathrm{min})\end{array}$ & $\begin{array}{l}q_{\mathrm{e}}(\mathrm{cal}) \\
(\mathrm{mg} / \mathrm{g})\end{array}$ & $R^{2}$ & $\begin{array}{c}k_{2} \\
(g /(m g m i n)\end{array}$ & $\begin{array}{c}q_{\mathrm{e}}(\mathrm{cal}) \\
(\mathrm{mg} / \mathrm{g})\end{array}$ & \\
\hline 25 & CAM & 2.2 & 0.006 & 1.5 & 89 & 0.012 & 2.3 & 0.99 \\
\hline 30 & CAM & & 0.00 & & 0.88 & 0.0 & 2.6 & 0.94 \\
\hline 40 & CAN & 2.5 & 0.004 & 14 & 0.84 & 9 & 2.6 & 0.99 \\
\hline 25 & CAY & 2.2 & 0.009 & 1.5 & 0.87 & 0.023 & 2.3 & 0.99 \\
\hline 30 & CAY & 2. & & & 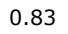 & & 2.5 & 0.99 \\
\hline 40 & CAY & .8 & 0.009 & .3 & 0.65 & 0.037 & 2.9 & 0.99 \\
\hline
\end{tabular}

T=Temperatura; AD: Adsorbente

Constantes PP: Constantes del modelo cinético de pseudo primer orden Constantes PS: Constantes del modelo cinético de pseudo segundo orden
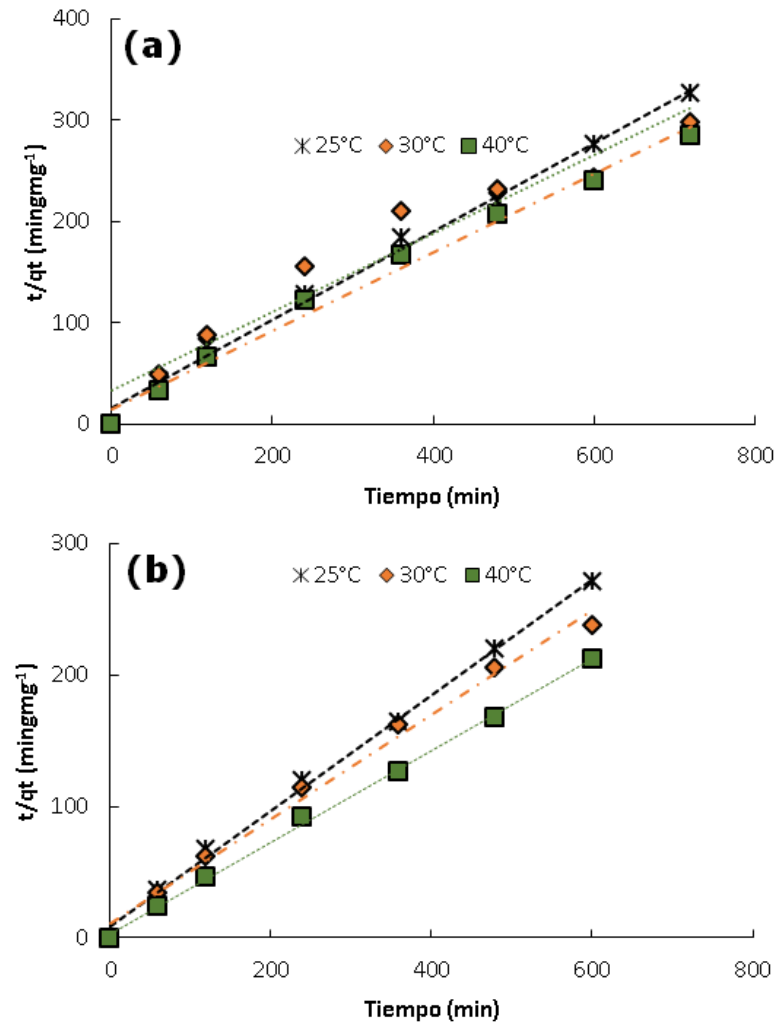

Figura 6. Modelo cinético pseudo segundo orden para la adsorción del colorante DB-86 a diferentes temperaturas: a) CAM y b) CAY.

\section{DISCUSIÓN}

La aplicación del carbón activado como material adsorbente está asociado a procesos de superficie: propiedades de textura y naturaleza química. Estos parámetros son intrínsecos del material de carbón, es decir, dependen de su origen y tratamientos encaminados a modificar su estructura porosa como también su química de superficie. En el caso de esta investigación el tratamiento de la cáscara de yuca con $\mathrm{H}_{3} \mathrm{PO}_{4}$ modificó ambas características; con respecto al área específica estimada a partir de los resultados del índice de yodo para ambos carbones activados, puede decirse que es baja con relación a las características genéricas de este tipo de adsorbentes, en consecuencia, la química de superficie desempeña un papel importante y quizás relevante. Factores como la presencia de heteroátomos principalmente aquellos grupos funcionales orgánicos tales como ácidos carboxílicos (también en la forma de su anhídrido cíclico), lactonas y grupos hidroxílicos de carácter fenólico (Tabla 1), son los responsables de las propiedades ácidas del carbón activado (23-25) y, la presencia de grupos pirona y cromero que le imprimen densidad electrónica sobre las capas grafénicas, especialmente de los electrones $n$ deslocalizados, son los responsables del carácter básico.

El carbón activado preparado a partir de cáscara de yuca presenta mayor contenido de grupos ácidos $(0.68$ $\mathrm{meq} / \mathrm{g}$ ), razón por la cual, su valor de $\mathrm{pH}$ en el punto de carga cero sea 3.1, característica que se esperaba porque fue activado con ácido fosfórico. Por otro lado, aunque no se conozca cómo fue activado el carbón 
comercial el hecho que tenga mayor desarrollo de meso y macroporosidad $\left(0.32 \mathrm{~cm}^{3} / \mathrm{g}\right)$ hace que su acidez disminuya (26).

Uno de los factores más importantes que afecta la capacidad de adsorbentes en el tratamiento de aguas residuales es el $\mathrm{pH}$. La Figura 3 muestra el efecto del $\mathrm{pH}$ en la remoción del colorante DB-86 en los dos carbones estudiados. El comportamiento es muy similar: la capacidad máxima de adsorción disminuye en la medida que aumenta el $\mathrm{pH}$. Estos cambios pueden atribuirse a (i) las interacciones entre los grupos funcionales superficiales del adsorbente y los grupos funcionales de las moléculas del colorante (grupos sulfonatos) variando la concentración de $\mathrm{H}^{+}$y $\mathrm{OH}^{-} \mathrm{y}$, (ii) las interacciones del adsorbente con otros grupos funcionales (enlaces de hidrógeno y grupos solubles en agua) (10).

En la explicación del posible efecto del $\mathrm{pH}$, la determinación del potencial de carga cero (PCC) juega un papel importante. Tomando como ejemplo el PCC del carbón activado CAY, para valores de $\mathrm{pH}$ por encima de 3.1 predominan las cargas negativas en la superficie del carbón. A valores de $\mathrm{pH}$ por debajo del PCC, la superficie presenta principalmente cargas positivas.

En el caso del colorante DB-86 al mezclarse en agua se disuelve instantáneamente disociándose los grupos sulfonatos como sigue:

$$
\mathrm{D}-\mathrm{SO}_{3} \mathrm{Na} \stackrel{\mathrm{H}_{2} \mathrm{O}}{\longrightarrow} \mathrm{D}^{-\mathrm{SO}_{3}^{-}}+\mathrm{Na}^{+}
$$

A pH 2 por debajo del PCC la superficie del carbón activado CAY se encuentra cargada positivamente:

$\mathrm{CAY} \stackrel{\mathrm{pH}<3.1}{\longrightarrow} \mathrm{CAY}^{+}$

Como resultado, el proceso de adsorción procede ampliamente a través de atracciones electrostáticas entre los dos contraiones:

$$
\mathrm{CAY}^{+}+\mathrm{D}-\mathrm{SO}_{3}^{-} \rightleftharpoons \mathrm{CAY}^{+-} \mathrm{O}_{3} \mathrm{~S}-\mathrm{D}
$$

Al incrementarse el $\mathrm{pH}$ de la solución $(\mathrm{pH}>3.1)$ la superficie del carbón activado se carga negativamente:

$\mathrm{CAY} \stackrel{\mathrm{pH}^{>} 3.1}{\longrightarrow} \mathrm{CAY}^{-}$

Como el colorante DB-86 sigue cargado negativamente por los grupos sulfonatos, las interacciones disminuyen, por lo tanto, baja su adsorción. El hecho que el carbón activado CAY siga removiendo aún a $\mathrm{pH}$ básicos se debe a fuerzas tipo van der Waals y enlaces de hidrógeno.

Los datos experimentales del estudio por lote se ajustaron a dos modelos de isotermas de adsorción: (i) el modelo de Langmuir se eligió para estimar la capacidad máxima de adsorción, los resultados del ajuste muestran que el carbón CAY obtuvo la mayor capacidad con $6.2 \mathrm{mg} / \mathrm{g}$ a $25^{\circ} \mathrm{C}$. Los coeficientes de correlación lineal considerablemente menores a 1 para todas las temperaturas estudiadas (Tabla 2), sugiere que el proceso de adsorción no ocurre en sitios activos idénticos localizados sobre la superficie de los diferentes carbones activados, en consecuencia, el proceso de adsorción no se restringe al cubrimiento en monocapas y, (ii) el modelo de Freundlich que consiste en una ecuación empírica que se puede usar para la adsorción no ideal sobre una superficie heterogénea; el parámetro $\mathrm{n}$ del modelo es una medida de la desviación de la linealidad de la adsorción. Si n es igual a la unidad, la adsorción es lineal. Si $n$ es inferior a la unidad, esto implica que el proceso de adsorción es químico, pero un valor de $\mathrm{n}$ superior a la unidad, la adsorción es favorable para un proceso físico, según las consideraciones de este modelo (27). En la tabla 2 se observa que los valores de $n$ son superiores a 1 ; esto sugiere que predomina la adsorción física.

Comparando los coeficientes de correlación de ambos modelos (Tabla 2), se observa que el modelo de isoterma de Freundlich es el que mejor representa el equilibrio de adsorción $\left(R^{2}>0.93\right)$ a todas las temperaturas evaluadas. Esto sugiere que el proceso de adsorción se lleva a cabo sobre una superficie heterogénea con crecimiento en multicapas.

Los valores negativos de $\Delta \mathrm{G}^{\circ}$ para ambos carbones activados demuestra que el proceso de adsorción del colorante DB-86 fue espontáneo. Resultados similares que muestran la espontaneidad del proceso de adsorción de colorantes han sido reportados por otros investigadores: Aljeboree et al (4), utilizaron la cáscara de coco como precursor en la preparación de carbón activado y su empleo en la remoción de colorantes textiles como el amarillo directo (DY12) y el azul maxilon (GRL); de la cáscara del fruto del pino brasileño, Calvete et al (28), prepararon carbón activado, que luego emplearon en la remoción del colorante reactivo naranja 16 (RO16); Leechart et al (29), usaron las cenizas del desecho del corte de la madera como material precursor en la preparación de carbón activado, para posteriormente evaluarlo en la remoción del colorante rojo reactivo 141 (RR141); entre otras investigaciones.

El cambio de entalpía positivo indica que la adsorción del colorante BD-86 es endotérmico. Cuando la atracción entre el colorante y el adsorbente tiene lugar, el cambio en la entalpía estándar ocurre por la presencia de fuerzas que incluyen van der Waals, enlaces de hidrógeno, intercambio de ligandos, interacciones dipolo-dipolo y enlaces químicos $(30,31)$. De acuerdo con la magnitud de la suma de las diferentes fuerzas la naturaleza de la adsorción puede ser física o química. Generalmente, la magnitud del cambio de entalpía estándar para la adsorción física está cercana a los $20 \mathrm{~kJ} / \mathrm{mol}$, mientras que la quimiadsorción está en el rango de los 80-200 $\mathrm{kJ} / \mathrm{mol}$ (32). En este estudio las entalpías calculadas corresponden a 39.5 y $60.5 \mathrm{~kJ} / \mathrm{mol}$ para los carbones activados CAM y CAY respectivamente, estos valores sugieren una tendencia hacia la fisiadsorción, debido a que el cambio entálpico está por debajo de lo requerido para que se presente quimiadsorción. Con respecto al cambio positivo en la entropía estándar se confirma la preferencia del colorante DB-86 sobre la superficie de los carbones activados evaluados.

Con respecto al ajuste de los datos experimentales a los modelos cinéticos, la ecuación de pseudo segundo orden muestra un excelente ajuste con un coeficiente de correlación lineal $\mathrm{R}^{2}>0.94$ para todas las condiciones de temperaturas estudiadas. De igual manera, los valores 
de $\mathrm{q}_{\mathrm{e}}$ calculados a partir del modelo cinético son muy similares a los obtenidos experimentalmente.

En conclusión, tanto el carbón activado obtenido a partir de la cáscara de yuca como el comercial tienen distinta composición química promovida muy seguramente por el uso de precursores diferentes, como en su método de activación. La diferencia entre los puntos de carga cero, propiedades de textura y la presencia de grupos funcionales orgánicos oxigenados, entre otras propiedades fisicoquímicas, así lo evidencian. El ajuste de los datos experimentales a los modelos de isotermas de Langmuir y Freundlich permitió establecer que el carbón activado CAY fue el que mayor capacidad de remoción mostró $(6.2 \mathrm{mg} / \mathrm{g})$. El modelo cinético que mejor se ajusta es el de pseudo segundo orden y los resultados termodinámicos demuestran la espontaneidad de la adsorción del colorante DB-86 sobre los dos carbones activados. El desarrollo de una buena área superficial unida a su naturaleza ácida permite que el carbón activado obtenido a partir de la cáscara de yuca pueda emplearse en la remoción del colorante DB-86.

\section{Conflicto de interés}

Los autores declaran no tener conflictos de interés.

\section{REFERENCIAS}

1. Anirudhan TS, Ramachandran M. Adsorptive removal of basic dyes from aqueous solutions by surfactant modified bentonite clay (organoclay): Kinetic and competitive adsorption isotherm. Process Saf Environ Prot. 2015; 95:215-225. https://doi.org/10.1016/j.psep.2015.03.003

2. Arica MY, Bayramoglu G. Polyaniline coated magnetic carboxymethylcellulose beads for selective removal of uranium ions from aqueous solution. J Radioanal Nucl Chem. 2016; 310(2):711-724. https://doi. org/10.1007/s10967-016-4828-z

3. Bayramoglu G, Akbulut A, Liman G, Arica MY. Removal of metal complexed azo dyes from aqueous solution using tris(2-aminoethyl) amine ligand modified magnetic $\mathrm{p}$ (GMA-EGDMA) cationic resin: Adsorption, isotherm and kinetic studies. Chem Eng Res Des. 2017; 124:85-97. https://doi. org/10.1016/j.cherd.2017.06.005

4. Aljeboree AM, Alshirifi AN, Alkaim AF. Kinetics and equilibrium study for the adsorption of textile dyes on coconut shell activated carbón. Arab J Chem. 2017; 10(Supl 2):S3381-S3393. https://doi. org/10.1016/j.arabjc.2014.01.020

5. Castellar G, Angulo E, Zambrano A, Charris D. Equilibrio de adsorción del colorante azul de metileno sobre carbón activado. Rev UDCA Act \& Div Cient. 2013; 16(1):263-271. https://revistas. udca.edu.co/index.php/ruadc/article/view/882

6. Chabane L, Cheknane B, Zermane F, Bouras $O$, Baudu M. Synthesis and characterization of reinforced hybridporous beads: application to the adsorption of malachitegreen in aqueous solution. Chem Eng Res Des. 2017; 120: 291-302. https:// doi.org/10.1016/j.cherd.2016.12.014

7. Sari AA, Muryanto ST, Hadibarata T. Development of bioreactor systems for decolorization of Reactive Green 19 using white rot fungus. Desalin Water Treat. 2016; 57(15):7029-7039. https://doi.org/ $\underline{10.1080 / 19443994.2015 .1012121}$
8. Mirzadeh SS, Khezri SM, Rezaei S, Forootanfar $H$, Mahvi AH, Faramarzi MA. Decolorization of two synthetic dyes using the purified laccase of Paraconiothyrium variabile immobilized on porous silica beads. J Environ Health Sci Eng. 2014; 12(6):1-9. https://doi.org/10.1186/2052$\underline{336 x-12-6}$

9. Tavengwa NT, Cukrowska E, Chimuka L. Synthesis, adsorption and selectivity studies of $\mathrm{N}$-propyl quaternized magnetic poly(4-vinylpyridine) for hexavalent chromium. Talanta. 2013; 116:670-677. https://doi.org/10.1016/j.talanta.2013.07.034

10. Kyzas GZ, Lazaridis NK, Mitropoulos A. Removal of dyes from aqueous solutions with untreated coffee residues as potential low-cost adsorbents: Equilibrium, reuse and thermodynamic approach. Chem Eng J. 2012; 189-190: 148-159. https://doi. org/10.1016/j.cej.2012.02.045

11. Ho YS, McKay G. Sorption of dyes and copper ions onto biosorbents. Process Biochem. 2003; 38(7):1047-1061. https://doi.org/10.1016/s00329592(02)00239-x

12. Gonçalves M, Guerreiro M, De Oliveira L, De Castro C. A friendly environmental material: iron oxide dispersed over activated carbon from coffee husk for organic pollutants removal. J Environ Manage. 2013; 127:206-211. https://doi.org/10.1016/j. jenvman.2013.05.017

13. Hu Z, Srinivasan MP. Preparation of high-surfacearea activated carbons from coconut shell. Microporous Mesoporous Mater. 1999; 27(1):11-18. https://doi.org/10.1016/s1387-1811(98)00183-8

14. Li G, Zhu W, Zhang C, Zhang S, Liu L, Zhu L, Zhao W. Effect of a magnetic field on the adsorptive removal of methylene blue onto wheat straw biochar. Bioresour Technol. 2016; 206:16-22. https://doi.org/10.1016/j.biortech.2015.12.087 
15. Sun L, Chen D, Wan S, Yu Z. Performance, kinetics, and equilibrium of methylene blue adsorption on biochar derived from eucalyptus saw dust modified with citric, tartaric, and acetic acids. Bioresour Technol. 2015; 198:300-308. https:// doi.org/10.1016/j.biortech.2015.09.026

16. Jung KW, Choi BH, Hwang MJ, Jeong TU, Ahn $\mathrm{KH}$. Fabrication of granular activated carbons derived from spent coffee grounds by entrapment in calcium alginate beads for adsorption of acid orange 7 and methylene blue. Bioresour Technol. 2016; 219:185-195. https://doi.org/10.1016/j. biortech.2016.07.098

17. Albis A, López AJ, Romero MC. Remoción de azul de metileno de soluciones acuosas utilizando cáscara de yuca (Manihot esculenta) modificada con ácido fosfórico. Prospectiva. 2017; 15(2):60-73. https:// doi.org/10.15665/rp.v15i2.777

18. Gonçalves R, Martins C, Mendes N, Farias L, Ferreira RC, Oliveira A, Oliveira M, Ilhéu R. Preparation of activated carbons from cocoa shells and siriguela seeds using $\mathrm{H} 3 \mathrm{PO} 4$ and $\mathrm{ZnCL} 2$ as activating agents for BSA and $\boldsymbol{\alpha}$-lactalbumin adsorption. Fuel Process Technol. 2014; 126:476-486. https://doi. org/10.1016/j.fuproc.2014.06.001

19. Boehm HP. Chemical identification of surface groups. Adv Catal. 1966; 16: 179-274. https:// doi.org/10.1016/S0360-0564(08)60354-5

20. Nunell GV, Fernández ME, Bonelli PR, Cukierman $A L$. Conversion of biomass from an invasive species into activated carbons for removal of nitrate from wastewater. Biomass Bioenerg. 2012; 44:87-95. https://doi.org/10.1016/j.biombioe.2012.05.001

21. Figueroa D, Moreno A, Hormaza A. Equilibrio, termodinámica y modelos cinéticos en la adsorción de Rojo 40 sobre tuza de maíz. Rev Ing Univ Medellín. 2015; 14(26):105-120. https://doi. org/10.22395/rium.v14n26a7

22. Konicki W, Aleksandrzak M, Mijowska E. Equilibrium, kinetic and thermodynamic studies on adsorption of cationic dyes from aqueous solutions using graphene oxide. Chem Eng Res Des. 2017; 123:3549. https://doi.org/10.1016/j.cherd.2017.03.036

23. Contescu A, Contescu C, Putyera K, Schwarz J. Surface acidity of carbons characterized by their continuous pK distribution and Böehm titration. Carbon 1997; 35(1):83-94. https://doi. org/10.1016/s0008-6223(96)00125-x

24. Valencia J, Castellar G. Predicción de las curvas de ruptura para la remoción de plomo (II) en disolución acuosa sobre carbón activado en una columna empacada. Rev Fac Ing Univ Antioquia. 2013; 66:141-158. http://aprendeenlinea.udea.edu.co/ revistas/index.php/ingenieria/article/view/15231
25. Maldonado-Hódar FJ, Morales-Torres S, PerezCardenas AF, Carrasco-Marín F. Química superficial de los materiales de carbón. Bol Grupo Español Carbón. 2011; 20:10-15. http://www.gecarbon. org/Boletines/articulos/boletinGEC 020 art.3.pdf

26. Rincón-Silva N, Ramirez-Gomez W, Mojica-Sánchez L, Blanco-Martínez D, Giraldo L, Moreno-Piraján J. Obtención de carbones activados a partir de semillas de eucalipto, por activación química con H3PO4. Caracterización y evaluación de la capacidad de absorción de fenol desde solución acuosa. Ingeniería y Competitividad. 2014; 16(1):207-219. https://doi.org/10.25100/iyc. v16i1.3725

27. Kumar PS, Ramalingam S, Senthamarai C, Niranjanaa M, Vijayalakshmi P, Sivanesan S. Adsorption of dye from aqueous solution by cashew nut shell: studies on equilibrium isotherm, kinetics and thermodynamics of interactions. Desalination. 2010; 261(1-2):52-60. https://doi.org/10.1016/j. desal.2010.05.032

28. Calvete T, Lima EC, Cardoso NF, Vaghetti JCP, Dias SLP, Pavan FA. Application of carbon adsorbents prepared from Brazilian-pine fruit shell for the removal of reactive orange 16 from aqueous solution: Kinetic, equilibrium, and thermodynamic studies. J Environ Manage. 2010; 91(8):1695-1706. https://doi.org/10.1016/j.jenvman.2010.03.013

29. Leechart P, Nakbanpote W, Thiravetyan P. Application of 'waste' wood-shaving bottom ash for adsorption of azo reactive dye. J Environ Manage. 2009; 90(2):912-920. https://doi.org/10.1016/j. jenvman.2008.02.005

30. Li Q, Yue QY, Su Y, Gao BY, Sun HJ. Equilibrium, thermodynamics and process design to minimize adsorbent amount for the adsorption of acid dyes onto cationic polymer-loaded bentonite. Chem Eng J. 2010; 158(3):489-497. https://doi. org/10.1016/j.cej.2010.01.033

31. Von Oepen B, Kördel W, Klein W. Sorption of nonpolar and polar compounds to soils: processes, measurements and experience with the applicability of the modified OECD-Guideline 106. Chemosphere. 1991; 22(3-4):285-304. https:// doi.org/10.1016/0045-6535(91)90318-8

32. Gu B, Schmitt J, Chen Z, Liang L, McCarthy JF. Adsorption and desorption of natural organic matter on iron oxide: mechanisms and models. Environ Sci Technol. 1994; 28(1):38-46. https:// doi.org/10.1021/es00050a007 